\title{
Panax notoginseng saponins and their applications in nervous system disorders: a narrative review
}

\author{
Jing $\mathrm{Qu}^{1}$, Na Xu ${ }^{1}$, Jianliang Zhang ${ }^{2}$, Xiaokun Geng ${ }^{1,3}$, Ruihua Zhang ${ }^{3}$ \\ ${ }^{1}$ China-America Institute of Neuroscience, Beijing Luhe Hospital, Capital Medical University, Beijing, China; ${ }^{2}$ Department of Neurobiology, Beijing \\ Institute of Brain Disorders, Capital Medical University, Key Laboratory for Neurodegenerative Disease of the Ministry of Education, Beijing \\ Key Laboratory of Neural Regeneration and Repairing, Beijing Key Laboratory of Brain Major Disorders-State Key Lab Incubation Base, Beijing \\ Neuroscience Disciplines, Beijing, China; ${ }^{3}$ Department of Neurology, Beijing Luhe Hospital, Capital Medical University, Beijing, China \\ Contributions: (I) Conception and design: R Zhang, J Qu; (II) Administrative support: X Geng, J Zhang; (III) Provision of study materials or patients: \\ R Zhang; (IV) Collection and assembly of data: J Qu, N Xu; (V) Data analysis and interpretation: R Zhang, J Qu; (VI) Manuscript writing: All \\ authors; (VII) Final approval of manuscript: All authors. \\ Correspondence to: Ruihua Zhang. Department of Neurology, Beijing Luhe Hospital, Capital Medical University, No. 82 Xinhua South Road, Beijing \\ 101149, China. Email: 15910380817@163.com.
}

\begin{abstract}
Panax notoginseng saponins (PNS), also called "sanqi" in Chinese, are the main active ingredients which are extracted from the root of Panax notoginseng (Burk.) F. H. Chen., and they have been traditionally used as a medicine in China for hundreds of years with magical medicinal value. PNS have varied biological functions, such as anti-inflammatory effects, anti-cancer effects, anti-neurotoxicity, and the prevention of diabetes. Nervous system disorders, a spectrum of diseases originating from the nervous system, have a significant impact on all aspects of patients' lives. Due to the dramatic gains in global life expectancy, the prevalence of nervous system disorders is growing gradually. Even if the mechanism of these diseases is still not clear, they are mainly characterized by neuronal dysfunction and neuronal death. Consequently, it is essential to find measures to slow down or prevent the onset of these diseases. At present, traditional Chinese medicines, as well as their active components, have gained widespread popularity in preventing and treating these diseases because of their merits, especially PNS. In this review, we predominantly address the recent advances in PNS researches and their biological functions, and highlight their applications in nervous system disorders, such as Alzheimer's disease (AD), Parkinson's disease (PD), and stroke.
\end{abstract}

Keywords: Panax notoginseng saponins (PNS); nervous system disorders; Alzheimer's disease (AD); Parkinson's disease (PD); stroke

Submitted Sep 10, 2020. Accepted for publication Nov 17, 2020.

doi: 10.21037/atm-20-6909

View this article at: http://dx.doi.org/10.21037/atm-20-6909

\section{Introduction}

Traditional Chinese medicine has gained widespread popularity in recent years. Furthermore, a myriad of studies have confirmed the effectiveness of these medicines for treating nervous system diseases. Among the wide variety of traditional Chinese medicines, Panax notoginseng (Burk.) F. H. Chen is one of the most commonly used products, and panax notoginseng saponins (PNS) are the main active compounds which are extracted from the root of Panax notoginseng. PNS contain about 20 different kinds of saponin constituents, among which ginsenoside $\mathrm{Rb} 1$, ginsenoside $\mathrm{Rg} 1$, notoginsenoside $\mathrm{R} 1$, ginsenoside $\mathrm{Rd}$, and ginsenoside Re are the top five saponins, which constitute up to $90 \%$ of total PNS $(1,2)$. Among these saponins, Rb1 and Rd are classified as protopanaxadioltype saponins (PDS), while Rg1, R1, and Re are classified as protopanaxatriol-type saponins (PTS) (3). Compared with other traditional Chinese medicine, PNS have more advantages, including long history and various 


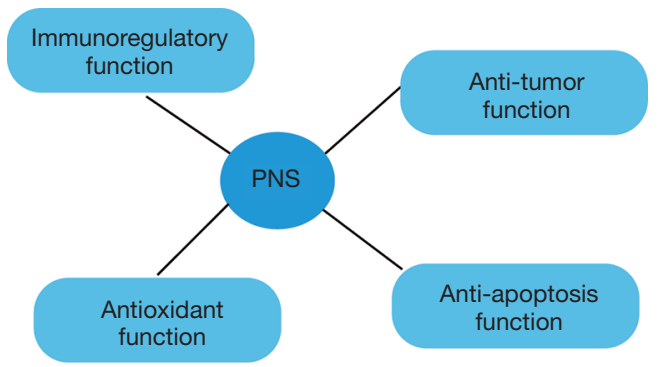

Figure 1 The biological functions of Panax notoginseng saponins (PNS), including immunoregulatory effects, antioxidant capacity, anti-apoptotic effects and anti-tumor properties.

pharmacological effects. Because these various beneficial effects, for example the inhibition of inflammatory responses, reduction of oxidative stress, and the inhibition of apoptosis, they are widely used therapeutically for the treatment of nervous system diseases.

Nervous system diseases, for example Alzheimer's disease (AD), Parkinson's disease (PD) and stroke, significantly disrupt patients' lives. Nowadays, the prevalence of nervous system disorders is growing, partly due to the dramatic increase in life expectancy. However, effective treatments are still lacking. Even if the etiology of these disorders remains elusive, they share common pathological features, including neuronal dysfunction and neuronal death. Therefore, it is urgent to seek novel approaches to slow down or prevent these diseases. In recent years, PNS have gained widespread popularity because of their various beneficial effects. As a kind of traditional Chinese medicines, the research hotspots and potential advantages of the pharmacological effects of PNS on nervous system diseases are the neuroprotection effects. This review provides an update on the applications of PNS in nervous system diseases. We present the following article in accordance with the Narrative Review reporting checklist (available at http://dx.doi.org/10.21037/ atm-20-6909).

\section{The biological functions of PNS}

\section{Immunoregulatory function}

The inflammatory response is a complex biological process which occurs when healthy tissues are invaded by physical stimuli, toxins, bacteria or viruses. It plays a significant role in the pathological processes of multiple diseases by releasing inflammatory cytokines, reactive oxygen free radicals, nuclear transcription factors, anti-inflammatory
Qu et al. PNS and their applications in nervous system disorders

neuropeptides and so on (4).

Previous studies have suggested an anti-inflammatory role of PNS both in vitro and in vivo (Figure 1). Rhule et al. found Rb1 suppressed the production of tumor necrosis factor- $\alpha$ (TNF- $\alpha$ ) and interleukin 6 (IL-6) induced by lipopolysaccharide (LPS) in cultured macrophages in a dose-dependent manner in vitro (5). Rh1 inhibited histamine release from mast cells and the IgE-mediated passive cutaneous anaphylaxis reaction in vivo (6). In addition, PNS modulated the proliferation and differentiation of Th17 cells by downregulating the levels of inflammatory cytokines and cell cycle genes (1). Hence, PNS could be potentially applied as an anti-inflammatory agent.

\section{Antioxidant function}

Oxidative stress is produced by a series of reactions caused by various stimuli, bacteria, viruses or toxins. It is related to an increase in reactive oxygen species production or a decrease in the effectiveness of antioxidant defenses (7). Oxidative stress from oxidative metabolism can cause base damage and strand breaks in DNA. Base damage is mainly caused by the generation of reactive oxygen species, such as $\mathrm{O}_{2}{ }^{-}, \mathrm{OH}^{-}$and $\mathrm{H}_{2} \mathrm{O}_{2}$. Some of these reactive oxygen species act as cellular messengers in redox signaling, hence, oxidative stress can cause disruptions to the normal mechanisms of cellular signaling, and lead to many pathophysiological conditions in the body, such as nervous system disorders (8).

PNS have been shown to have protective properties via enhancing antioxidant enzyme activity, such as catalase (CAT), superoxide dismutase (SOD), and glutathione peroxidase (GSHPX) in senescence-accelerated mouseprone 8 (SAMP8) mice and mouse melanoma B16 cells $(9,10)$. In addition, PNS had protective effects on oxidative stress-induced brain cell damage, which was associated with a reduction in reactive oxygen species (ROS) levels and an upregulation in antioxidant mediators, such as heme oxygenase-1 (HO-1) and glutathione S-transferase pi 1 (11). Furthermore, Rb1 protected cells from oxidative injury induced by $\mathrm{H}_{2} \mathrm{O}_{2}$, which was related to inhibiting endoplasmic reticulum stress (12). Hence, PNS could be potentially applied as an antioxidative agent.

\section{Anti-apoptotic function}

Apoptosis, or programmed cell death, is a mechanism which occurs in multicellular organisms when stimulated 
by various triggers (13). Apoptotic cell death is a neat, orderly process. The characteristics of apoptosis include a reduction in the volume of cells and their nuclei, the loss of connections to adjacent cells, the formation of blebs on the cell surface, the dissection of chromatin into fragments, and the rapid engulfment of the cell corpse by phagocytosis. It is well known that the progress of apoptosis can be regulated by the expression of B-cell lymphoma protein 2 (Bcl-2, an anti-apoptotic gene) and Bcl-2 associated $\mathrm{X}$ protein (Bax, a pro-apoptotic gene) (14). Caspase-3, downstream to Bcl-2 and Bax, also plays a key role in the execution phase of apoptosis (15). Apoptosis has recently been the focus of investigations into the mechanisms underlying multisystem diseases.

Cao et al. found PNS could suppress the abnormal apoptosis and autophagy of hippocampal neurons in mice with learning and memory impairment, which was associated by re-activating phosphoinositide 3-kinase/Akt/mammalian target of rapamycin signaling transduction (16). Yuan et al. found that PNS had a protective effect against avascular necrosis of the femoral head induced by steroids through the inhibition of apoptosis and caspase- 3 activation (17). In addition, PNS also reversed decreases in Bcl-2 expression and increases in Bax and caspase- 3 expression induced by $\mathrm{H}_{2} \mathrm{O}_{2}$ in bone marrow stromal cells (18). Hence, PNS could be potentially used as an anti-apoptotic agent.

\section{Anti-tumor functions}

Tumor, or cancer, involves abnormal cell growth of tissue with the potential to spread or invade to other healthy parts of the body (19). The possible signs and symptoms of cancer include the formation of a lump, abnormal bleeding, prolonged cough, unexplained weight loss and a change in bowel movements. Presently, over 100 types of cancers can affect human beings, highlighting it is a serious threat to human health. In recent years, traditional Chinese medicines have caught people's attention to treat cancers, including PNS.

PNS was able to halt SW480 human colorectal cancer cells in the $\mathrm{S}$ and G2/M phases (20). Moreover, PNS showed an anti-proliferative effect in hepatoma Hep3B cells, which was indicated by reduced tumor volume and weight (21). PNS had an inhibitory effect on metastatic breast carcinoma cell line 4T1 migration and invasion (22). PNS could also attenuate lung cancer growth by modulating the protein levels of Met/miR-222 axis (23). Furthermore, R1 inhibited human colorectal cancer HCT116 cell metastasis by inhibiting cell migration, adhesion, and invasion through regulating the expression of metastasis-associated signaling molecules (24). Hence, PNS could be potentially applied as an anti-tumor agent.

In summary, PNS have a wide range of functions, such as anti-inflammatory, anti-oxidative, anti-apoptotic, and antitumor effects.

\section{The applications of PNS in nervous system diseases}

Nervous system diseases encompass over 600 different disorders, from neurodegenerative diseases such as $\mathrm{AD}$ and PD, to stroke. Along with an aging society, these diseases have attracted worldwide medical attention because of their widespread prevalence. Therefore, it is urgent to find novel approaches to slow down or prevent these diseases. Recently, traditional Chinese medicines, including PNS, have attracted worldwide attention because of their clinical efficacy in a wide spectrum of diseases, including nervous system diseases.

\section{PNS and AD}

$\mathrm{AD}$, characterized by progressive cognitive impairment and memory impairment, is a neurodegenerative disease affecting 30 million patients worldwide. Along with the general increase in human life span, it is gradually becoming a heavy burden and challenge. The main hypotheses of the pathogenesis of $\mathrm{AD}$ are cholinergic theory and $\beta$-amyloid peptide $(\mathrm{A} \beta)$ theory $(25,26)$. Treatment of $\mathrm{AD}$ is targeted towards the formation and deposition of $A \beta$, tau protein phosphorylation, and the cholinergic system.

\section{Effects of PNS on A $\beta$ formation and deposition}

Amyloid precursor protein (APP) is widely found in the cell membrane of many tissues. Normal APP is shredded by $\alpha$-secretase to produce soluble APP, which is important for cell nutrition. In addition, APP is cleaved by -secretase and $\gamma$-secretase to produce $\mathrm{A} \beta$, which mainly exists in two forms-A $\beta 1-40$ and $A \beta 1-42$. A $\beta 1-42$ is easily oligomerized, which has a toxic effect on neurons. A small amount of $A \beta$ can also be degraded by extracellular enzymes, including neprilysin (NEP) and insulin degrading enzyme (IDE).

Studies show that PNS can downregulate the expression of the APP gene in the brain, upregulate the expression of ADAM9 mRNA, then promote APP to be shredded by $\alpha$-secretase and inhibit the cleavage of APP by $\beta$-site 


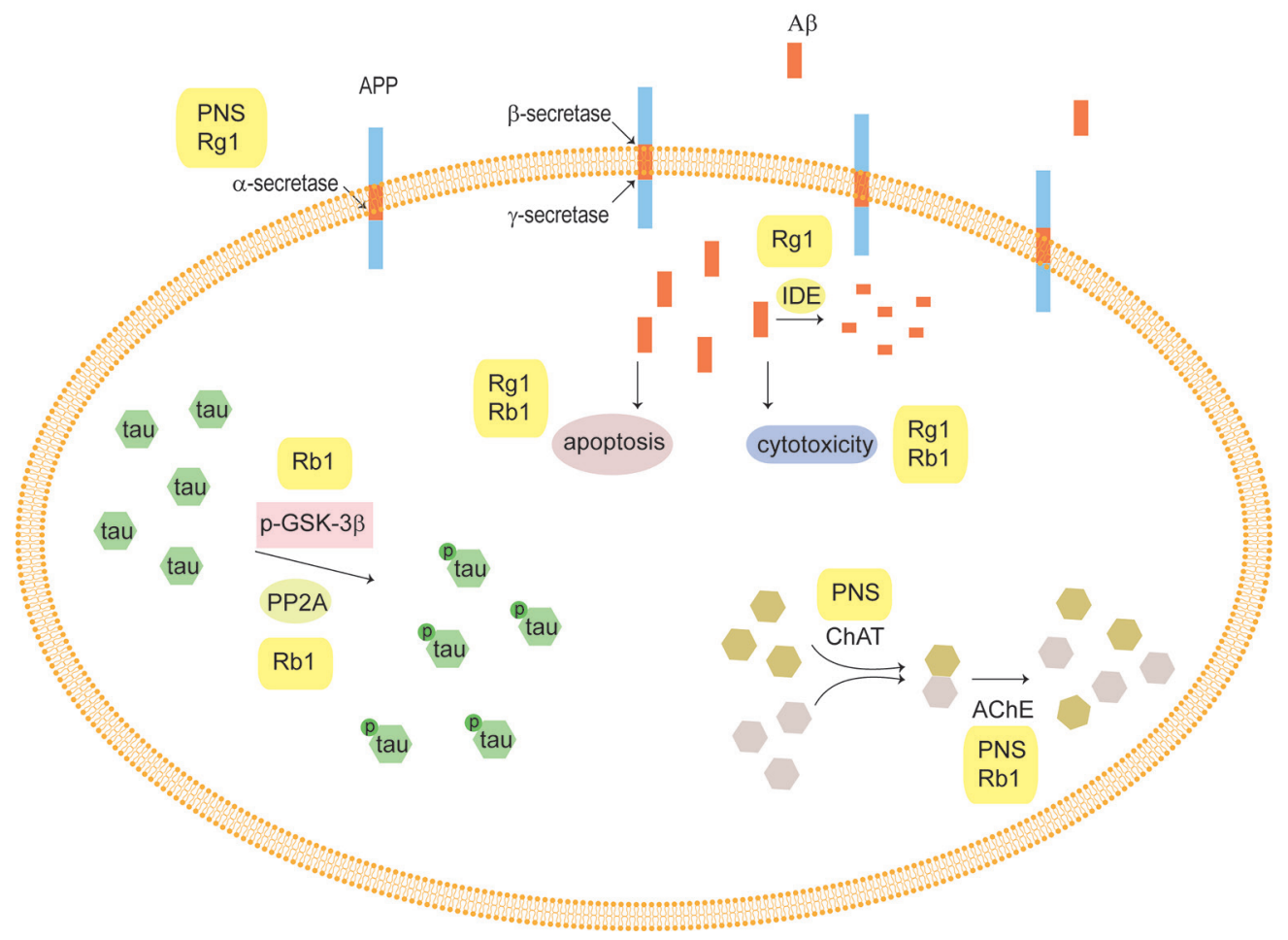

Figure 2 Panax notoginseng saponins (PNS) and Alzheimer's disease (AD). Amyloid precursor protein (APP) is shredded by $\alpha$-secretase to produce soluble APP, and cleaved by $\beta$-secretase and $\gamma$-secretase to produce $\beta$-amyloid (A $\beta$ ) which has toxic effects on neurons. PNS could promote APP shearing by $\alpha$-secretase, and attenuate the cytotoxicity of neurons induced by A $\beta$. The hyperphosphorylation of tau plays a key role in the pathogenesis of AD. PNS can regulate $\mathrm{p}-\mathrm{GSK}-3 \beta$ and PP2A levels to prevent tau hyperphosphorylation. Acetylcholine (ACh) is an important neurotransmitter which promotes learning and improves memory ability. PNS can increase the content and activity of choline acetyltransferase (ChAT), and inhibit acetylcholinesterase (AChE) activity.

amyloid precursor protein cleaving enzyme 1 (BACE1). This downregulated the production of $\mathrm{A} \beta$, which ameliorated learning and memory ability in mice (27) (Figure 2). More precisely, $\mathrm{Rg} 1$ increased the expression of ADAM10 mRNA and promoted APP shearing by $\alpha$-secretase (28), and inhibited APP sheared by $\beta$-secretase and $\gamma$-secretase (29), which decreased the production of BACE1. Rg1 also activated peroxisome proliferator-activated receptor gamma $(\operatorname{PPAR} \gamma)$ to upregulate the expression of IDE and enhance $\mathrm{A} \beta$ degradation in an $\mathrm{AD}$ rat model (30).

Wu et al. found that $\mathrm{Rg} 1$ could attenuate the cytotoxicity of neurons induced by A $\beta 25-35$ and protect primary rat cerebrocortical neurons by upregulating ERK1/2 phosphorylation and reducing nuclear factor kappa-lightchain-enhancer of activated B cells $(\mathrm{NF}-\kappa \mathrm{B})$ nuclear translocation (31) (Figure 2). In addition, they also found $\operatorname{Rg} 1$ could inhibit the apoptosis induced by $A \beta 25-$ 35 in human endothelial cells through downregulation of the tyrosine nitration initiated by hypoxia-inducible transcription factor 1 alpha (HIF-1 $\alpha$ ), and inhibiting mitochondrial apoptosis (32).

In addition, $\mathrm{Rb} 1$ could inhibit the production of ROS, increase $\mathrm{Bcl}-2 / \mathrm{Bax}$ ratio and decrease caspase-3 activity, which resisted cell damage induced by A 3 (33) (Figure 2).

\section{Effects of PNS on the phosphorylation of tau protein}

Tau protein is a microtubule-associated protein, and its hyperphosphorylation performs central roles in the pathogenic mechanisms of AD (34). The high phosphorylation of tau protein is associated with glycogen synthase kinase-3 (GSK-3) and protein phosphatase 2A (PP2A) (35).

It was found that $\mathrm{Rb} 1$ could protect mice against toxicity induced by a neurotoxin through blocking the hyperphosphorylation of tau via regulating the levels of p-GSK-3 $\beta$ and PP2A. Therefore, Rb1 might be used as a 


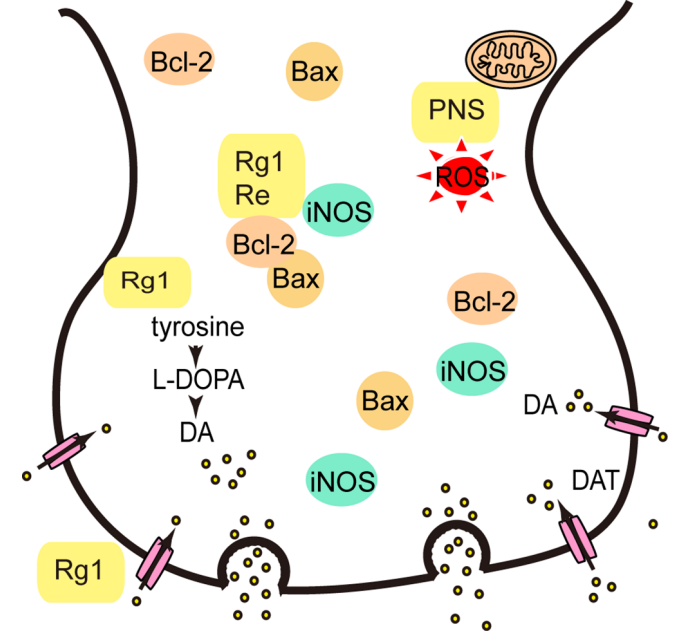

Figure 3 Panax notoginseng saponins (PNS) and Parkinson's disease (PD). PNS can inhibit neurotoxicity through enhancing antioxidant activity [reactive oxygen species (ROS), inducible nitric oxide synthase (iNOS)], modulating inflammation, and inhibiting mitochondria-mediated apoptosis (Bcl-2, Bax). PNS demonstrates neuroprotective effects on dopaminergic neurons by regulating tyrosine hydroxylase (TH), and dopamine transporter (DAT).

potential preventive drug candidate for $\mathrm{AD}$ as well as other tau pathology-associated disorders (36,37) (Figure 2).

\section{Effect of PNS on acetylcholine}

Acetylcholine (ACh) is one of the important neurotransmitters in the central cholinergic system, and its main function is to promote learning and improve memory ability. ACh is synthesized by choline acetyltransferase (ChAT), and decomposed by acetylcholinesterase (AChE). The balance of ACh is maintained by ChAT and AChE (38). If the activities of ChAT and AChE are abnormal, it will cause the decompensation of ACh, which leads to biochemical changes in the central cholinergic system.

PNS have a strong protective effect on cholinergic neurons in $\mathrm{AD}$ rat models (Figure 2). PNS can repair and improve damaged neurons to increase the quantity and quality of surviving cells, and increase the content and activity of ChAT, which protects and improves the function of the central cholinergic system.

Previous studies have demonstrated that both Rb1 and $\mathrm{Rg} 1$ can alleviate cognitive deficits, and are also effective in boosting neuron survival in the spinal cord. Furthermore, Wang et al. compared the effects of $\mathrm{Rg} 1$ and $\mathrm{Rb} 1$ on dementia in a mouse model. They found that both Rb1 and $\operatorname{Rg} 1$ ameliorated cognitive impairment using stepdown passive avoidance (SD) and Morris water maze (MWM) tests, thereby increasing the levels of ACh in the hippocampus. However, they found $\operatorname{Rg} 1$ inhibited the activity of AChE while Rb1 had no effect on AChE activity. In addition, both $\mathrm{Rb} 1$ and $\mathrm{Rg} 1$ also prevented a decrease in 5-HT caused by scopolamine, but Rb1 was more active than $\operatorname{Rg} 1$ at the same dose (39).

In conclusion, PNS have been demonstrated to inhibit $A \beta$ formation and deposition, prevent tau hyperphosphorylation, and maintain ACh balance, suggesting that PNS might be a potential treatment strategy for $\mathrm{AD}$ (Figure 2).

\section{PNS and PD}

$\mathrm{PD}$, the second most common neurodegenerative disorder, affects $1 \%$ of individuals over 60 years old. It is defined by symptoms of motor impairment, including resting tremor, postural instability, bradykinesia, and rigidity. $\mathrm{PD}$ is characterized by the selective loss of dopaminergic neurons in the substantia nigra pars compacta (40). At present, 6-hydroxydopamine (6-OHDA), 1-Methy-4phenyl-1,2,3,6-tetrahydropyridine (MPTP) and its active metabolite 1-methyl-4-phenylpyridinium ion $\left(\mathrm{MPP}^{+}\right)$are used in models to induce PD.

Luo et al. found that PNS increased the expression of thioredoxin-1 (Trx-1) and attenuated $\mathrm{MPP}^{+}$-induced neurotoxicity in vitro (41) (Figure 3). Subsequently, they found PNS inhibited neurotoxicity induced by MPTP in vivo through enhancing antioxidant activity, modulating inflammation and inhibiting mitochondria-mediated apoptosis (42). Rb1 had neuroprotective effects on dopaminergic neurons via stimulating estrogen receptors with consequent activation of ERK1/2 and Akt, as well as inhibiting the levels of SAPK/JNK and p38 (43). Apart from $\mathrm{Rb} 1, \mathrm{Rg} 1$ also had neuroprotective effects on dopaminergic cells in a rat model of PD induced by 6-OHDA, which might be associated with the activation of the insulin-like growth factor receptor (IGF-IR) pathway. The authors found that $\mathrm{Rg} 1$ significantly antagonized the decrease in tyrosine hydroxylase (TH), dopamine transporter (DAT), and Bcl-2 in the substantia nigra induced by 6-OHDA (44). Re prevented apoptosis of substantia nigra neurons in a mouse model of PD induced by MPTP, which could be related to the upregulation of Bcl-2, the downregulation of Bax and inducible nitric oxide synthase (iNOS) levels, and the inhibition of caspase-3 activation (45). 


\section{Page 6 of 10}

Overall, PNS have demonstrated neuroprotective effects on dopaminergic cells, which suggests that PNS might be a potential treatment strategy for PD (Figure 3).

\section{PNS and stroke}

Cerebral ischemia is characterized by ischemia and hypoxia of brain tissue caused by cerebral blood flow supply. If cerebral ischemia is not completely blocked for 0.5 hours, cerebral blood flow will return to normal again, and will lead to ischemia-reperfusion injury (46). The treatment of cerebral ischemia mainly involves protecting neurons, reducing the infarct size, and decreasing neuronal death $(46,47)$. Ischemic stroke, especially permanent occlusions, accounts for the overwhelming majority of strokes worldwide. Apart from artery occlusion, apoptosis, inflammatory responses, oxidative stress, angiogenesis, and neural plasticity play pivotal roles in the severity of cerebral ischemia injury and clinical prognosis.

PNS have been shown to reduce infarct volumes and neurological damage in a rat model of middle cerebral artery occlusion (MCAO) by downregulating the expression of TNF- $\alpha$ and IL-1 $\beta$, upregulating the expression of IL-10 (2), attenuating TUNEL-positive cells, and decreasing the levels of caspase- 1 and caspase-3 (48). Moreover, PNS were able to enhance angiogenesis in ischemic boundary zones, increase capillary densities, and increase the expression of vascular endothelial growth factor (VEGF) as well as angiopoietin-1 (Ang-1) (49). In addition, PNS also promoted stroke recovery by downregulating the expression of neural plasticity-associated proteins, such as nogo-A, NgR and neurotrophic factor, ROCK2, in MCAO rats and in SH-SY5Y cells induced by oxygen-glucose deprivation/reperfusion (OGD/R) (50,51).

$\mathrm{Rb} 1$ induced neuroprotection in rats with cerebral ischemia by inhibiting the gene levels of TNF- $\alpha$, IL- $1 \beta$, IL-6, as well as the activation of the NF- $\mathrm{BB}$ pathway, and increasing the level of brain-derived neurotrophic factor (BDNF) (52,53). Additionally, Liang et al. found that after Rb1 treatment, SH-SY5Y cells resisted apoptosis induced by $\mathrm{OGD} / \mathrm{R}$, which was associated with the protection of mitochondrial function through inhibiting the release of apoptosis inducing factor (AIF) and cytochrome c (Cytc) (54).

$\mathrm{Rg} 1$ also had a protective effect on ischemiareperfusion injury which was related to inhibiting the apoptosis of hippocampal neurons, and regulating the expression of phospho-JunN-terminal kinase (p-JNK) and p-ERK1/2 (30). Additionally, R1 had protective effects on
Qu et al. PNS and their applications in nervous system disorders

injury induced by cerebral ischemia-reperfusion in vivo and in vitro. Its mechanism was associated with the activation of the Akt/Nrf2 pathway to inhibit the activity of NADPH oxidase and the defunctionalization of mitochondria (55).

In brief, PNS have been demonstrated to reduce infarct volumes and neurological damage, as well as regulate neural plasticity, suggesting that PNS shows potential as a treatment strategy for stroke (Figure 4).

\section{PNS and other nervous system diseases}

The N-methyl-D-aspartic acid (NMDA) receptor can regulate neuronal survival, dendrite and axon structural development, synaptic plasticity, neuronal circuit formation, and learning and memory activity $(3,56)$. An imbalance in NMDA receptor activity may contribute to the development of nervous system diseases $(57,58)$. R1 was shown to have a protective effect on mouse neurons exposed to glutamate (Glu) in vitro by acting on NMDA receptors, which indicated $\mathrm{R} 1$ might be a candidate in patients who were diagnosed neurodegenerative diseases related with Glu excitotoxicity (59).

Neurotrophic factors are proteins which play key roles in the development, survival and apoptosis of neurons. Nerve growth factor (NGF), an important neurotrophic factor, is a potential drug target for the treatment of nerve injury. $\mathrm{Rb} 1$ and $\mathrm{Rg} 1$ have been shown to increase the expression of NGF in Schwann cells, the glial cells in the peripheral nervous system, which suggests they could exert neuroprotective effects in peripheral nerve injuries (60).

Microglia, the brain's innate immune cells, can transition to an activated phenotype during an inflammatory response. Reactive microglia not only protect neurons, but also secrete cytotoxic factors to damage neurons. The activation of microglia plays an important role in nervous system diseases $(61,62)$. PNS have been shown to have an inhibitory effect on hippocampal microglia activation in SAMP8 mice, as $\mathrm{Rg} 1$ inhibited the activation of microglia by activating the PLC- $\gamma$ signaling pathway (63).

Ion channels, including sodium $\left(\mathrm{Na}^{+}\right)$, potassium $\left(\mathrm{K}^{+}\right)$, calcium $\left(\mathrm{Ca}^{2+}\right)$, play important roles in nervous system disorders. PNS could significantly inhibit ion channel currents in a dose- and voltage-dependent manner $(64,65)$. It can be inferred PNS might be useful agents for regulation of ion channel current, which could be used for the treatment of neuron system diseases. And the neuroprotection mechanism of PNS is via the mechanism of regulating channels and receptors $(66,67)$. 


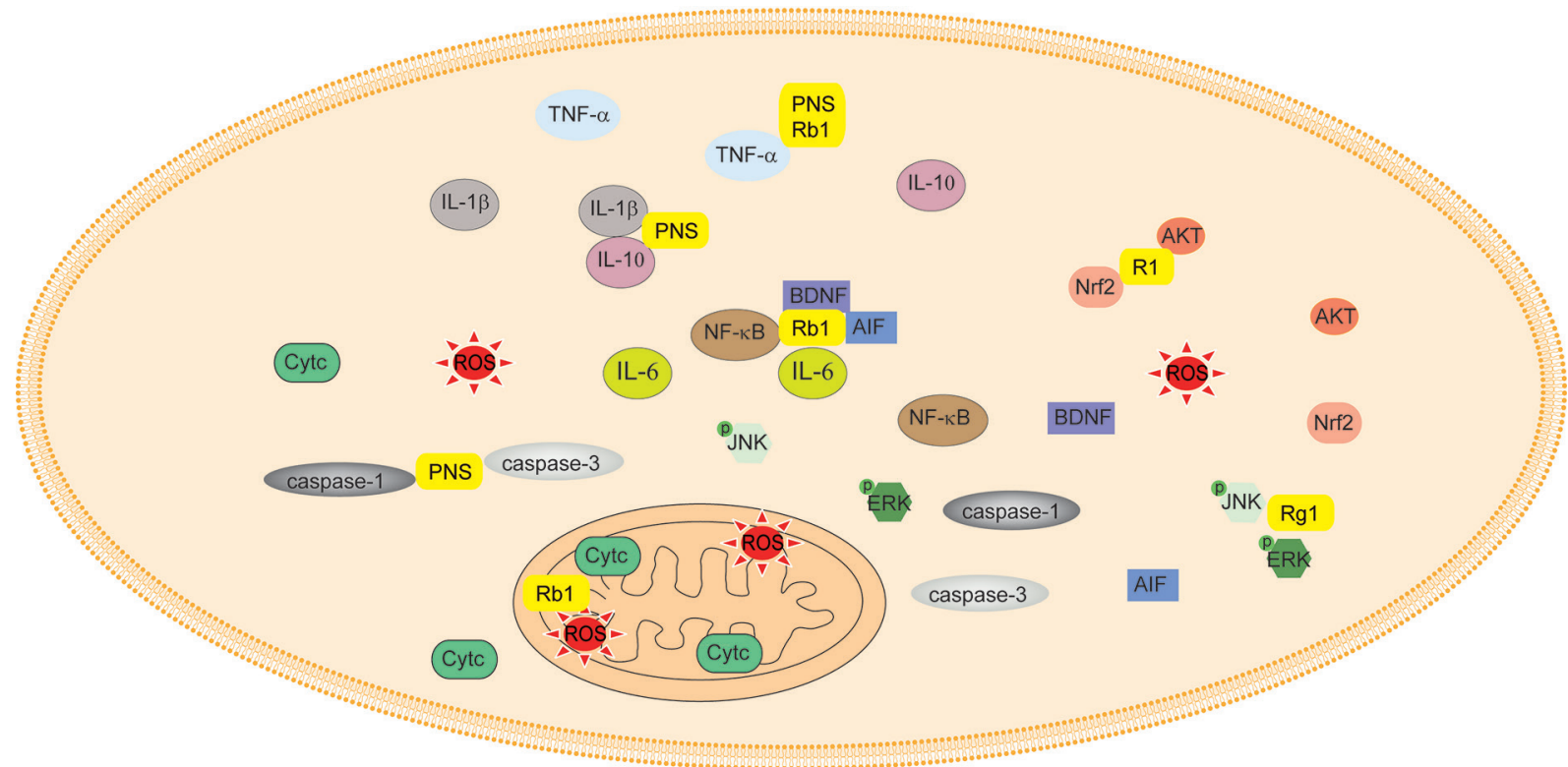

Figure 4 Panax notoginseng saponins (PNS) and stroke. PNS had protective effects on ischemia-reperfusion injury by regulating the genes involved in the inflammatory response, including TNF- $\alpha$, IL-1 $\beta$, IL-6, and IL-10, and apoptosis-related genes caspase-1, caspase-3, as well as p-JNK, p-ERK, Akt, Nrf2, AIF and Cytc.

\section{Conclusions and perspectives}

In recent years, PNS have become one of the most popular healthcare products, especially for many older adults who have hypertension, hyperlipidemia, hyperglycemia, and nervous system diseases. PNS contain about 20 different kinds of saponin constituents, among which Rb1, Rg1, $\mathrm{R} 1, \mathrm{Rd}$ and $\mathrm{Re}$ are the top five saponins, which constitute up to $90 \%$ of total PNS. In this review, we focused on the significant roles of the main components of PNS on the prevention and treatment of nervous system diseases.

The bioactive components of PNS can slow vascular aging through anti-inflammatory, anti-oxidative, antiapoptotic, and anti-tumor effects. Due to these merits, PNS exert protective effects on numerous diseases, such as nervous system disorders, cancer (68), coronary artery diseases (69), hyperlipidemia and obesity (70), osteoarthritic chondrocytes (71) and diabetes (72). With a dramatic increase in life expectancy, most countries have an aging society, meaning that nervous system disorders, including neurodegenerative disorders, have attracted worldwide attention. Therefore, it is urgent to find novel approaches to prevent or slow down these nervous system disorders. Based on demonstrated findings, traditional Chinese medicines, especially PNS, are promising natural agents highly needed as alternatives effective and safe for the management of nervous system disorders, such as $\mathrm{AD}, \mathrm{PD}$ and stroke, which suggests PNS might be potential neuroprotective drugs. However, the effects of PNS on peripheral nervous system diseases are not clear. The clinical applications and research value of PNS are of great significance, however, the mechanisms underlying its various therapeutic effects need to be further studied.

\section{Acknowledgments}

Funding: The Science and Technology Plan of Beijing Tongzhou District (KJ2019CX001-23, KJ2020CX006-08).

\section{Footnote}

Reporting Checklist: The authors have completed the Narrative review reporting checklist. Available at http:// dx.doi.org/10.21037/atm-20-6909

Conflicts of Interest: All authors have completed the ICMJE uniform disclosure form (available at http://dx.doi. org/10.21037/atm-20-6909). The authors have no conflicts of interest to declare.

Ethical Statement: The authors are accountable for all 


\section{Page 8 of 10}

aspects of the work in ensuring that questions related to the accuracy or integrity of any part of the work are appropriately investigated and resolved.

Open Access Statement: This is an Open Access article distributed in accordance with the Creative Commons Attribution-NonCommercial-NoDerivs 4.0 International License (CC BY-NC-ND 4.0), which permits the noncommercial replication and distribution of the article with the strict proviso that no changes or edits are made and the original work is properly cited (including links to both the formal publication through the relevant DOI and the license). See: https://creativecommons.org/licenses/by-nc-nd/4.0/.

\section{References}

1. Wei JR, Wen X, Bible PW, et al. Panax Notoginseng Saponin Controls IL-17 Expression in Helper T Cells. J Ocul Pharmacol Ther 2017;33:285-9.

2. Shi X, Yu W, Liu L, et al. Panax notoginseng saponins administration modulates pro- /anti-inflammatory factor expression and improves neurologic outcome following permanent MCAO in rats. Metab Brain Dis 2017;32:221-33.

3. Zhao H, Han Z, Li G, et al. Therapeutic Potential and Cellular Mechanisms of Panax Notoginseng on Prevention of Aging and Cell Senescence-Associated Diseases. Aging Dis 2017;8:721-39.

4. Pavlov VA, Chavan SS, Tracey KJ. Molecular and Functional Neuroscience in Immunity. Annu Rev Immunol 2018;36:783-812.

5. Rhule A, Navarro S, Smith JR, et al. Panax notoginseng attenuates LPS-induced pro-inflammatory mediators in RAW264.7 cells. J Ethnopharmacol 2006;106:121-8.

6. Park EK, Choo MK, Han MJ, et al. Ginsenoside Rh1 possesses antiallergic and anti-inflammatory activities. Int Arch Allergy Immunol 2004;133:113-20.

7. Sies H, Berndt C, Jones DP. Oxidative Stress. Annu Rev Biochem 2017;86:715-48.

8. Simpson DSA, Oliver PL. ROS Generation in Microglia: Understanding Oxidative Stress and Inflammation in Neurodegenerative Disease. Antioxidants (Basel) 2020;9:743.

9. Huang JL, Jing X, Tian X, et al. Neuroprotective Properties of Panax notoginseng Saponins via Preventing Oxidative Stress Injury in SAMP8 Mice. Evid Based Complement Alternat Med 2017;2017:8713561.

10. Peiran L, Ying L, Mingzhuo Z, et al. The development of a Panax notoginseng medicinal liquor processing
Qu et al. PNS and their applications in nervous system disorders

technology using the response surface method and a study of its antioxidant activity and its effects on mouse melanoma B16 cells. Food Funct 2017;8:4251-64.

11. Zhou N, Tang Y, Keep RF, et al. Antioxidative effects of Panax notoginseng saponins in brain cells. Phytomedicine 2014;21:1189-95.

12. Zeng XS, Jia JJ, Ma LF. Gensenoside Rb1 protects rat PC12 cells from oxidative stress-induced endoplasmic reticulum stress: the involvement of thioredoxin-1. Mol Cell Biochem 2015;410:239-46.

13. Bedoui S, Herold MJ, Strasser A. Emerging connectivity of programmed cell death pathways and its physiological implications. Nat Rev Mol Cell Biol 2020;21:678-95.

14. Singh R, Letai A, Sarosiek K. Regulation of apoptosis in health and disease: the balancing act of BCL-2 family proteins. Nat Rev Mol Cell Biol 2019;20:175-93.

15. Danial NN, Korsmeyer SJ. Cell death: critical control points. Cell 2004;116:205-19.

16. Cao Y, Yang Y, Wu H, et al. Stem-leaf saponins from Panax notoginseng counteract aberrant autophagy and apoptosis in hippocampal neurons of mice with cognitive impairment induced by sleep deprivation. J Ginseng Res 2020;44:442-52.

17. Yuan HF, Pan JF, Li S, et al. Protective effects of total saponins of panax notoginseng on steroid-induced avascular necrosis of the femoral head in vivo and in vitro. Evid Based Complement Alternat Med 2015;2015:165679.

18. Qiang H, Zhang C, Shi ZB, et al. Protective effects and mechanism of Panax Notoginseng saponins on oxidative stress-induced damage and apoptosis of rabbit bone marrow stromal cells. Chin J Integr Med 2010;16:525-30.

19. Hayes JD, Dinkova-Kostova AT, Tew KD. Oxidative Stress in Cancer. Cancer Cell 2020;38:167-97.

20. Wang CZ, Xie JT, Zhang B, et al. Chemopreventive effects of Panax notoginseng and its major constituents on SW480 human colorectal cancer cells. Int J Oncol 2007;31:1149-56.

21. Lin YW, Mou YC, Su CC, et al. Antihepatocarcinoma activity of lactic acid bacteria fermented Panax notoginseng. J Agric Food Chem 2010;58:8528-34.

22. Wang P, Cui J, Du X, et al. Panax notoginseng saponins (PNS) inhibits breast cancer metastasis. J Ethnopharmacol 2014;154:663-71.

23. Yang $Q$, Wang $P$, Cui J, et al. Panax notoginseng saponins attenuate lung cancer growth in part through modulating the level of Met/miR-222 axis. J Ethnopharmacol 2016;193:255-65.

24. Lee CY, Hsieh SL, Hsieh S, et al. Inhibition of human colorectal cancer metastasis by notoginsenoside $\mathrm{R} 1$, an 
important compound from Panax notoginseng. Oncol Rep 2017;37:399-407.

25. Busche MA, Hyman BT. Synergy between amyloidbeta and tau in Alzheimer's disease. Nat Neurosci 2020;23:1183-93.

26. van der Kant R, Goldstein LSB, Ossenkoppele R. Amyloid-beta-independent regulators of tau pathology in Alzheimer disease. Nat Rev Neurosci 2020;21:21-35.

27. Huang J, Wu D, Wang J, et al. Effects of Panax notoginseng saponin on alpha, beta, and gamma secretase involved in Abeta deposition in SAMP8 mice. Neuroreport 2014;25:89-93.

28. Shi C, Na N, Zhu X, et al. Estrogenic effect of ginsenoside Rg1 on APP processing in post-menopausal platelets. Platelets 2013;24:51-62.

29. Fang F, Chen X, Huang T, et al. Multi-faced neuroprotective effects of Ginsenoside $\mathrm{Rg} 1$ in an Alzheimer mouse model. Biochim Biophys Acta 2012;1822:286-92.

30. Quan Q, Wang J, Li X, et al. Ginsenoside Rg1 decreases Abeta(1-42) level by upregulating PPARgamma and IDE expression in the hippocampus of a rat model of Alzheimer's disease. PLoS One 2013;8:e59155.

31. Wu J, Pan Z, Wang Z, et al. Ginsenoside Rg1 protection against beta-amyloid peptide-induced neuronal apoptosis via estrogen receptor alpha and glucocorticoid receptor-dependent anti-protein nitration pathway. Neuropharmacology 2012;63:349-61.

32. Yan J, Liu Q, Dou Y, et al. Activating glucocorticoid receptor-ERK signaling pathway contributes to ginsenoside $\mathrm{Rg} 1$ protection against beta-amyloid peptide-induced human endothelial cells apoptosis. J Ethnopharmacol 2013;147:456-66.

33. Xie X, Wang HT, Li CL, et al. Ginsenoside Rb1 protects PC12 cells against beta-amyloid-induced cell injury. Mol Med Rep 2010;3:635-9.

34. Drummond E, Pires G, MacMurray C, et al. Phosphorylated tau interactome in the human Alzheimer's disease brain. Brain 2020;143:2803-17.

35. Martin L, Page G, Terro F. Tau phosphorylation and neuronal apoptosis induced by the blockade of PP2A preferentially involve GSK3beta. Neurochem Int 2011;59:235-50.

36. Zhao HH, Di J, Liu WS, et al. Involvement of GSK3 and PP2A in ginsenoside Rb1's attenuation of aluminuminduced tau hyperphosphorylation. Behav Brain Res 2013;241:228-34.

37. Zhao R, Zhang Z, Song Y, et al. Implication of phosphatidylinositol-3 kinase/Akt/glycogen synthase kinase-3 beta pathway in ginsenoside Rb1's attenuation of beta-amyloid-induced neurotoxicity and tau phosphorylation. J Ethnopharmacol 2011;133:1109-16.

38. Hampel H, Mesulam MM, Cuello AC, et al. The cholinergic system in the pathophysiology and treatment of Alzheimer's disease. Brain 2018;141:1917-33.

39. Wang Q, Sun LH, Jia W, et al. Comparison of ginsenosides $\mathrm{Rg} 1$ and $\mathrm{Rb} 1$ for their effects on improving scopolamine-induced learning and memory impairment in mice. Phytother Res 2010;24:1748-54.

40. Poewe W, Seppi K, Tanner CM, et al. Parkinson disease. Nat Rev Dis Primers 2017;3:17013.

41. Luo FC, Wang SD, Li K, et al. Panaxatriol saponins extracted from Panax notoginseng induces thioredoxin-1 and prevents 1-methyl-4-phenylpyridinium ion-induced neurotoxicity. J Ethnopharmacol 2010;127:419-23.

42. Luo FC, Wang SD, Qi L, et al. Protective effect of panaxatriol saponins extracted from Panax notoginseng against MPTP-induced neurotoxicity in vivo. $\mathrm{J}$ Ethnopharmacol 2011;133:448-53.

43. Hashimoto R, Yu J, Koizumi H, et al. Ginsenoside Rb1 Prevents MPP(+)-Induced Apoptosis in PC12 Cells by Stimulating Estrogen Receptors with Consequent Activation of ERK1/2, Akt and Inhibition of SAPK/ JNK, p38 MAPK. Evid Based Complement Alternat Med 2012;2012:693717.

44. Xu L, Chen WF, Wong MS. Ginsenoside Rg1 protects dopaminergic neurons in a rat model of Parkinson's disease through the IGF-I receptor signalling pathway. $\mathrm{Br}$ J Pharmacol 2009;158:738-48.

45. Xu BB, Liu CQ, Gao X, et al. Possible mechanisms of the protection of ginsenoside Re against MPTP-induced apoptosis in substantia nigra neurons of Parkinson's disease mouse model. J Asian Nat Prod Res 2005;7:215-24.

46. Campbell BCV, Khatri P. Stroke. Lancet 2020;396:129-42.

47. Chamorro Á, Dirnagl U, Urra X, et al. Neuroprotection in acute stroke: targeting excitotoxicity, oxidative and nitrosative stress, and inflammation. Lancet Neurol 2016;15:869-81.

48. Li H, Deng CQ, Chen BY, et al. Total saponins of Panax notoginseng modulate the expression of caspases and attenuate apoptosis in rats following focal cerebral ischemia-reperfusion. J Ethnopharmacol 2009;121:412-8.

49. Hui Z, Sha DJ, Wang SL, et al. Panaxatriol saponins promotes angiogenesis and enhances cerebral perfusion after ischemic stroke in rats. BMC Complement Altern Med 2017;17:70. 


\section{Page 10 of 10}

50. Liu L, Zhu L, Zou Y, et al. Panax notoginseng saponins promotes stroke recovery by influencing expression of Nogo-A, NgR and p75NGF, in vitro and in vivo. Biol Pharm Bull 2014;37:560-8.

51. Shi X, Yu W, Yang T, et al. Panax notoginseng saponins provide neuroprotection by regulating NgR1/RhoA/ ROCK2 pathway expression, in vitro and in vivo. $\mathrm{J}$ Ethnopharmacol 2016;190:301-12.

52. Zhu J, Jiang Y, Wu L, et al. Suppression of local inflammation contributes to the neuroprotective effect of ginsenoside $\mathrm{Rb} 1$ in rats with cerebral ischemia. Neuroscience 2012;202:342-51.

53. Jiang Z, Wang Y, Zhang X, et al. Preventive and therapeutic effects of ginsenoside Rb1 for neural injury during cerebral infarction in rats. Am J Chin Med 2013;41:341-52.

54. Liang J, Yu Y, Wang B, et al. Ginsenoside Rb1 attenuates oxygen-glucose deprivation-induced apoptosis in SH-SY5Y cells via protection of mitochondria and inhibition of AIF and cytochrome c release. Molecules 2013;18:12777-92.

55. Meng X, Wang M, Wang X, et al. Suppression of NADPH oxidase- and mitochondrion-derived superoxide by Notoginsenoside R1 protects against cerebral ischemiareperfusion injury through estrogen receptor-dependent activation of Akt/Nrf2 pathways. Free Radic Res 2014;48:823-38.

56. Zhou Q, Sheng M. NMDA receptors in nervous system diseases. Neuropharmacology 2013;74:69-75.

57. Wang R, Reddy PH. Role of Glutamate and NMDA Receptors in Alzheimer's Disease. J Alzheimers Dis 2017;57:1041-8.

58. Gonzalez J, Jurado-Coronel JC, Avila MF, et al. NMDARs in neurological diseases: a potential therapeutic target. Int J Neurosci 2015;125:315-27.

59. Gu B, Nakamichi N, Zhang WS, et al. Possible protection by notoginsenoside $\mathrm{R} 1$ against glutamate neurotoxicity mediated by N-methyl-D-aspartate receptors composed of an NR1/NR2B subunit assembly. J Neurosci Res 2009;87:2145-56.

60. Liang W, Ge S, Yang L, et al. Ginsenosides Rb1 and Rg1 promote proliferation and expression of neurotrophic factors in primary Schwann cell cultures. Brain Res 2010;1357:19-25.

61. Yates D. Modulating microglia. Nat Rev Neurosci 2018;19:387.

62. Navarro V, Sanchez-Mejias E, Jimenez S, et al. Microglia in Alzheimer's Disease: Activated, Dysfunctional or Degenerative. Front Aging Neurosci 2018;10:140.
Qu et al. PNS and their applications in nervous system disorders

63. Zong Y, Ai QL, Zhong LM, et al. Ginsenoside Rg1 attenuates lipopolysaccharide-induced inflammatory responses via the phospholipase $\mathrm{C}$-gamma1 signaling pathway in murine BV-2 microglial cells. Curr Med Chem 2012;19:770-9.

64. Kang DI, Lee JY, Yang JY, et al. Evidence that the tertiary structure of 20(S)-ginsenoside $\operatorname{Rg}(3)$ with tight hydrophobic packing near the chiral center is important for $\mathrm{Na}(+)$ channel regulation. Biochem Biophys Res Commun 2005;333:1194-201.

65. Lee BH, Lee JH, Yoon IS, et al. Mutations of arginine 222 in pre-transmembrane domain I of mouse 5-HT(3A) receptor abolish 20(R)- but not 20(S)-ginsenoside $\operatorname{Rg}(3)$ inhibition of 5-HT-mediated ion currents. Biol Pharm Bull 2007;30:1721-6.

66. Kang MS, Baek SH, Chun YS, et al. Modulation of lipid kinase PI4KIIalpha activity and lipid raft association of presenilin 1 underlies gamma-secretase inhibition by ginsenoside (20S)-Rg3. J Biol Chem 2013;288:20868-82.

67. Park SM, Choi MS, Sohn NW, et al. Ginsenoside Rg3 attenuates microglia activation following systemic lipopolysaccharide treatment in mice. Biol Pharm Bull 2012;35:1546-52.

68. Guo YH, Kuruganti R, Gao Y. Recent Advances in Ginsenosides as Potential Therapeutics Against Breast Cancer. Curr Top Med Chem 2019;19:2334-47.

69. Duan L, Xiong X, Hu J, et al. Panax notoginseng Saponins for Treating Coronary Artery Disease: A Functional and Mechanistic Overview. Front Pharmacol 2017;8:702.

70. Wang Q, Mu RF, Liu X, et al. Steaming Changes the Composition of Saponins of Panax notoginseng (Burk.)

F.H. Chen That Function in Treatment of Hyperlipidemia and Obesity. J Agric Food Chem 2020;68:4865-75.

71. Zhang Y, Cai W, Han G, et al. Panax notoginseng saponins prevent senescence and inhibit apoptosis by regulating the PI3KAKTmTOR pathway in osteoarthritic chondrocytes. Int J Mol Med 2020;45:1225-36.

72. Zhou P, Xie W, He S, et al. Ginsenoside Rb1 as an AntiDiabetic Agent and Its Underlying Mechanism Analysis. Cells 2019;8:204.

(English Language Editor: C. Betlazar-Maseh)

Cite this article as: Qu J, Xu N, Zhang J, Geng X, Zhang R. Panax notoginseng saponins and their applications in nervous system disorders: a narrative review. Ann Transl Med 2020;8(22):1525. doi: 10.21037/atm-20-6909 